\title{
Comparaison de mesures d'intensité énergétique dans les pêcheries commerciales
}

\author{
Pascal Le Floc' $h^{1}$, Isabelle Dangeard ${ }^{2}$ \\ 1 Économiste, Université de Brest, UEB, UMR AMURE, 29238 Brest cedex 3, France \\ ${ }^{2}$ Gestionnaire, Université de Brest, UEB, ERCS EREID, 29334 Quimper cedex, France
}

La pêche maritime professionnelle est à la fois un secteur économique en difficulté et une activité dont les conséquences environnementales sont pointées du doigt. L'originalité de cet article est de nous proposer, en se limitant à la Bretagne, une analyse de la consommation énergétique en fonction des pratiques de pêche et du type de navire. Les auteurs nous montrent que les politiques publiques n'ont pas toujours l'effet escompté.

La Rédaction

\section{Mots-clés :}

pêcheries ;

indicateurs ;

intensité énergétique ; gaz à effet de serre ; $\mathrm{CO}_{2}$

\section{Keywords:}

fisheries;

indicators; energy intensity; greenhouse gases; $\mathrm{CO}_{2}$

\begin{abstract}
Résumé - Les indicateurs écosystémiques ont pour fonction essentielle de nourrir le débat sur les moyens les plus efficaces dans la lutte contre le réchauffement climatique. L'activité de la pêche n'échappe pas à ce débat. Les évaluations engagées dans la filière des produits marins démontrent que l'utilisation de gazole pour la pêche a l'impact environnemental le plus important. La problématique soulevée à partir des estimations obtenues dans le cadre de quelques pêcheries françaises concerne la pertinence de cet indicateur d'intensité énergétique. On relève des mesures d'intensité énergétique plus fortes sur des cas d'étude locaux, tandis que des évaluations à plus grande échelle aboutissent à des résultats nettement inférieurs. Se pose dès lors la question de la pertinence d'indicateurs globaux.
\end{abstract}

\begin{abstract}
Comparing energy intensity results for commercial fisheries. The main objectives assigned to ecosystem indices are to fuel the debate on the best ways to reduce the impact of global warming. Fishing activities are also concerned with the debate. The assessments carried out about fish products show that the use of fuel in the fishing stage has the strongest impact on the environment. This paper addresses the issue of the significance of energy intensity from results obtained for French fishing fleets. Compared with measures from other international studies, the results show to be lower when the scale of analysis is large. Quantifying energy intensity on a global scale for fisheries could then be called into question.
\end{abstract}

\section{Introduction}

La production d'indicateurs écosystémiques ${ }^{1}$ est fortement encouragée dans le cadre de la Convention sur la diversité biologique (CDB), ratifiée en 1992 lors de la conférence de Rio. C'est aussi lors du deuxième Sommet de la Terre qu'a été adopté le texte de la Conventioncadre des Nations unies sur les changements climatiques (CCNUCC). Cette dernière part du constat que les activités humaines, principalement dans les pays développés, augmentent la concentration de gaz à effet de serre dans l'atmosphère terrestre, ce qui entraîne un réchauffement climatique. L'objectif de la Convention est donc de

Auteur correspondant : P. Le Floc'h, plefloch@univ-brest.fr

${ }^{1}$ Cet article fait suite à la présentation d'un poster lors de la conférence internationale de l'économie des pêches (IIFET) en 2010 à Montpellier. «stabiliser [... ] les concentrations de gaz à effet de serre [...] à un niveau qui empêche toute perturbation anthropique dangereuse du système climatique » (Nations unies, 1992). Entrée en vigueur en mars 1994, la CCNUCC énonce des objectifs qualitatifs sur lesquels les pays l'ayant ratifiée s'engagent. En 1997, le Protocole de Kyoto y a été ajouté, il donne des objectifs chiffrés de réduction des émissions de gaz à effet de serre, par pays, pour la période 2008-2012. Il est entré en vigueur en février 2005.

La pêche comprend différentes phases successives : capture, premier marché au débarquement ${ }^{2}$, transformation à terre par les entreprises de mareyage et par des unités spécialisées, marché de gros, transport, commerce de détail, consommation. Les analyses de cycle de vie

\footnotetext{
${ }^{2}$ Il s'agit souvent de ventes aux enchères dans les criées.
} 
Tableau 1. Caractéristiques moyennes des flottilles de Bretagne sud en 2005.

\begin{tabular}{|l|c|c|c|c|c|c|}
\hline & \multicolumn{2}{|c|}{ Dormants } & \multicolumn{4}{c|}{ Traînants } \\
\hline Classes de longueur (mètre) & $<12 \mathrm{~m}$ & $>12 \mathrm{~m}$ & $<12 \mathrm{~m}$ & $12-16 \mathrm{~m}$ & $16-20 \mathrm{~m}$ & $20-25 \mathrm{~m}$ \\
\hline Effectif & 43 & 6 & 8 & 28 & 13 & 31 \\
\hline Âge (année) & 19 & 27 & 18 & 23 & 23 & 18 \\
\hline Puissance motrice $(\mathrm{kW})$ & 129 & 175 & 128 & 220 & 302 & 433 \\
\hline
\end{tabular}

réalisées par Thrane (2006) et par Ellingsen et Aanondsen (2006) comparent, tout au long de ces étapes, des impacts environnementaux tels que la consommation d'énergies fossiles et le réchauffement climatique, la pollution des milieux, l'acidification et l'eutrophisation, mais excluent l'épuisement de la ressource halieutique et les dégâts collatéraux sur les écosystèmes. La pondération relative des différents types d'impacts environnementaux, qui n'apparaît pas explicitement dans ces études, amène ces auteurs à conclure sur l'importance prépondérante des impacts de la phase de capture. D'un point de vue exclusivement climatique, l'ADEME (2007) estime que l'impact le plus important est dû à la phase de capture et ajoute aux gaz à effet de serre émis par la pêche « 10 \% pour tenir compte du transport, de la manutention, de la chaîne du froid, etc. ». Ces constatations amènent certains auteurs (Schau et al., 2009; Schau et Magerholm Fet, 2008) à étudier exclusivement la phase de capture.

Pour évaluer l'impact environnemental de cette phase de capture, un indice d'intensité énergétique a été développé. Il mesure la consommation de carburant rapportée à la production en volume ou en valeur. Les estimations obtenues dans le cadre de quelques pêcheries françaises tendent néanmoins à montrer que les valeurs de cet indicateur d'intensité énergétique peuvent être affectées lors d'un changement d'échelle, d'un niveau fin sur des données individuelles à un niveau agrégé. En effet, la comparaison des mesures d'intensité énergétique à l'échelle de plusieurs pêcheries européennes confirme une divergence des valeurs obtenues pour cet indicateur selon le niveau d'agrégation. Dans cet article, nous analyserons l'évolution de l'intensité énergétique des pêches pour la flotte de Bretagne sud en distinguant différentes techniques de pêche et différents types de navires, avant de comparer ces résultats avec des données obtenues à des échelles plus globales. Cette flotte correspond aux 1830 navires présents dans la base de données comptable de l'Observatoire économique régional des pêches de Bretagne ${ }^{3}$, pris en compte sur la période 1994-2005.

\footnotetext{
3 À l'échelle bretonne, les données économiques de la pêche sont collectées par voie d'enquête auprès des pêcheurs et par voie comptable auprès des centres de gestion de la pêche artisanale. L'Ifremer, responsable du système d'information halieutique (SIH), gère l'ensemble des travaux d'enquête portant sur l'activité des navires, leurs productions par espèce et les données économiques. La couverture statistique ne se limite pas à la Bretagne, mais s'étend aux trois façades maritimes françaises
}

Les navires de pêche pour lesquels les données comptables et de production sont disponibles sont immatriculés dans les sept quartiers maritimes de Bretagne sud (Douarnenez, Audierne, Le Guilvinec, Concarneau, Lorient, Auray et Vannes). Cet échantillon de navires représente $9 \%$ de la population d'unités immatriculées en Bretagne (Observatoire économique régional des pêches de Bretagne, 2007).

\section{Données et méthodes}

\section{Caractéristiques de l'échantillon observé}

La typologie des navires de pêche tient compte de deux critères principaux : la technique de capture et la longueur du navire, et s'inspire des travaux engagés dans le cadre des programmes communautaires de collecte des données dans le secteur de la pêche (Communauté européenne, 2000 et 2001). On distingue les navires pratiquant les arts «traînants » (chalutage et drague principalement) des unités exerçant les arts «dormants » (filets, casiers, lignes). Appliquée aux navires de pêche de Bretagne sud, cette typologie selon le type de pêche et la longueur conduit à six catégories (Tab. 1).

Les navires pratiquant les arts " traînants » sont essentiellement des chalutiers (traction par l'arrière d'un filet en forme de poche), associant dans certains cas à la technique du chalut celle de la drague (traction par l'arrière ou le côté du navire d'un outil à armature métallique qui permet de racler les fonds marins pour la capture des coquillages, par exemple). Les unités de pêche de type «dormant » exploitent les stocks de poissons ou crustacés à l'aide de filets, de casiers ou de lignes, sans traction. La distinction principale porte sur la mobilité de l'engin de pêche, les techniques «traînantes » (chalut et drague) étant par définition plus mobiles que les techniques de production dites «dormantes ». Par conséquent, les moyens d'exploitation tractés sont plus intensifs en énergie. Les navires utilisant la senne ou la bolinche posent, au regard des objectifs de cette recherche,

(Manche-mer du Nord, Atlantique et Méditerranée). La donnée de type comptable est transmise par les centres de gestion à l'Observatoire économique régional des pêches de Bretagne, dirigé par une organisation professionnelle à laquelle sont associés l'UMR AMURE (www.umr-amure.fr) ainsi que le Comité régional des pêches de Bretagne. 

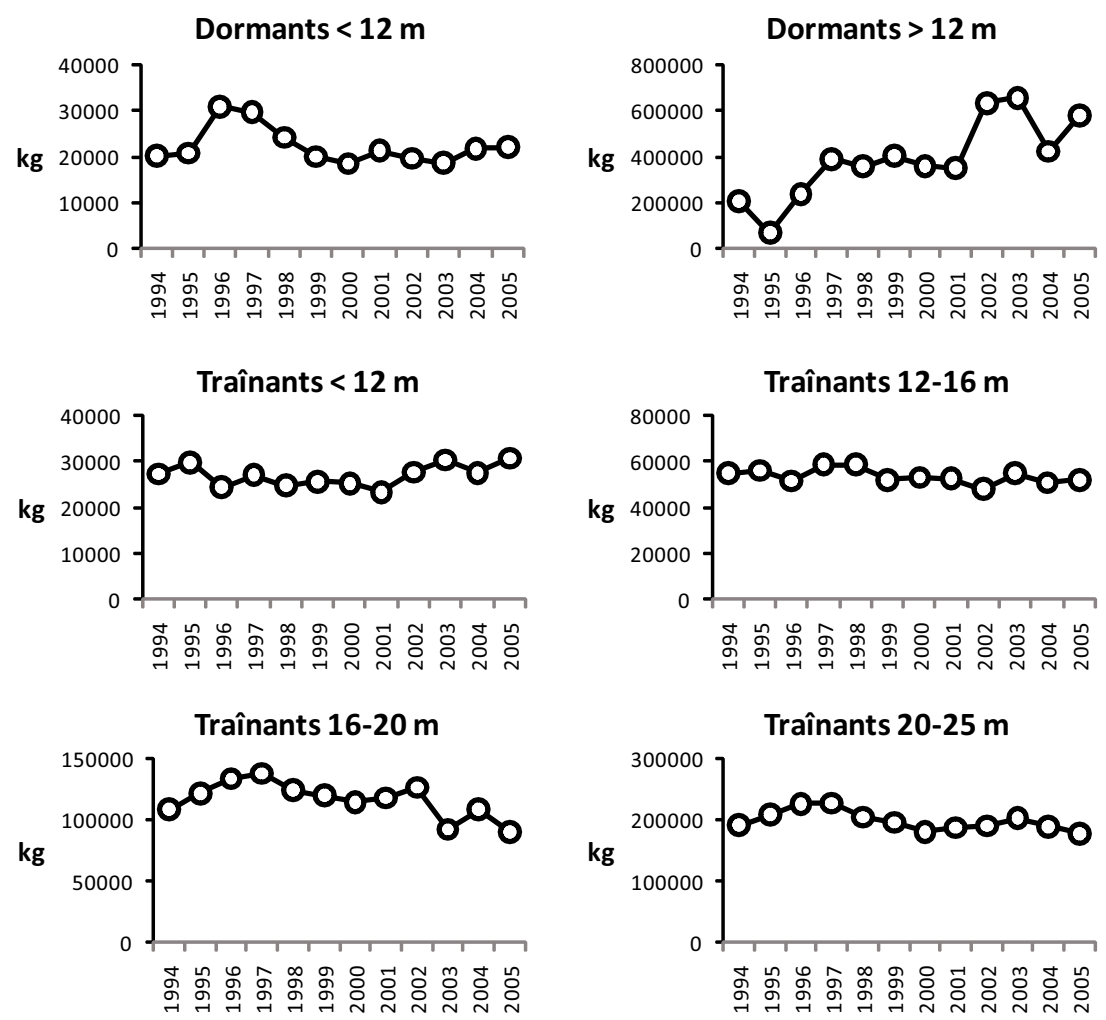

Fig. 1. Évolution des débarquements (kg) moyens par navire (flottilles de Bretagne sud) de 1994 à 2005.

un problème particulier. Ils sont classés avec les engins mobiles dans la typologie européenne, mais isolés des deux classes «traînant » et « dormant » dans la typologie adoptée par l'Ifremer pour les flottes françaises. Plutôt que d'en faire une catégorie particulière, nous avons décidé d'agréger leurs résultats avec ceux des navires dormants dans la mesure où leur consommation énergétique les rapproche plus de ces derniers.

L'effectif des différentes catégories est variable selon les années et a tendance à diminuer (31 unités recensées en 2005 dans la catégorie "traînants de plus de 20 mètres » alors que l'effectif atteignait 62 navires en 1994). On dispose pour chaque navire retenu dans l'analyse d'informations détaillées sur les espèces débarquées et la consommation énergétique.

L'évolution des débarquements est décrite par la figure 1. Les pêcheries (ou zones de pêche) exploitées par les dormants (moins et plus de 12 mètres) et les traînants de moins de 12 mètres se situent essentiellement dans la bande côtière (soit à 12 milles nautiques $^{4}$ au plus du trait de côte), dans le nord du golfe de Gascogne. Les traînants de plus de 12 mètres fréquentent des pêcheries principalement hors de la bande côtière du sud de la Bretagne au sud de l'Irlande. On relève une certaine stabilité de la production des navires dormants de moins de 12 mètres (de 18 à 30 tonnes d'espèces commerciales débarquées par navire) tandis que les dormants de plus de

\footnotetext{
4 Un mille nautique équivaut à 1852 mètres.
}

12 mètres enregistrent une forte augmentation des prises au cours de la période. D'un peu plus de 200 tonnes en 1994, leurs débarquements atteignent en moyenne 500 à 650 tonnes en 2005. Si les débarquements ont triplé, cette forte augmentation n'est que partiellement liée à un gain de productivité. Un changement structurel dans la composition de l'échantillon explique aussi ce niveau élevé des débarquements. Cette flottille affiche en 2005 les performances les plus élevées (mesurées par deux indicateurs de productivité apparente du travail et du capital) comparativement aux autres catégories (Le Floc'h et al., 2008a). Les écarts de production entre les quatre catégories de navires traînants vont de 1 à 8 entre les plus petites unités (longueur inférieure à 12 mètres) et les plus grandes (20-25 mètres), avec une stabilité relative des productions débarquées par navire au cours de la période d'étude.

Une différence significative entre dormants et traînants tient dans la variabilité des productions individuelles. Alors que les navires traînants ont des niveaux de débarquement proches à l'intérieur d'une même classe de longueur (le coefficient de variation est inférieur à 0,5 ), la quantité de biomasse débarquée par les navires dormants fluctue fortement selon les unités (le coefficient de variation est compris entre 0,6 et 1 selon les années). Cette variabilité témoigne d'une plus grande hétérogénéité des stratégies d'exploitation des stocks de poissons 
au sein des entreprises de pêche utilisant les techniques dormantes.

\section{Indicateurs d'intensité énergétique et émissions de gaz à effet de serre : méthodes de calcul}

Nous considérons comme source principale d'énergie le carburant utilisé pour la pêche. Celui-ci doit remplir deux fonctions : le déplacement entre le port de débarquement et les zones de production ou pêcheries (fonction essentiellement de transport) ; la recherche du poisson sur zone (fonction d'exploitation). Certaines modélisations bioéconomiques distinguent ces deux activités dans la détermination du coût de production (Sampson, 1990). L'indicateur d'intensité énergétique de la pêche représente la consommation unitaire de carburant pendant la phase de capture.

Nous estimons d'abord l'intensité énergétique de la pêche à partir d'un indicateur de consommation unitaire de carburant, égal à la quantité de carburant par unité de production, celle-ci étant mesurée soit en volume $(\mathrm{kg}$ de poisson), soit en valeur (€). La consommation unitaire de carburant doit ensuite être convertie en émission de gaz à effet de serre, pour permettre la comparaison avec les données de l'ADEME (2007) relatives au poisson et à la viande, exprimées en équivalent carbone ${ }^{5}$. Nous prendrons ici un facteur de conversion du gazole égal à $2,662 \mathrm{~kg}$ e- $\mathrm{CO}_{2}$ par litre, ou $0,726 \mathrm{~kg}$ e-C par litre.

$C^{\prime}$ est à partir de ces chiffres que l'ADEME a produit une estimation du facteur d'émission du poisson, à l'exclusion du thon et des crevettes, dont le mode de pêche conduit à des émissions plus importantes : "L'IFREMER indique que la pêche française consomme environ 250 millions de litres de diesel par an, pour environ 500000 tonnes de poissons débarqués chaque année. En première approximation, l'émission liée à la pêche d'une tonne de poisson sera donc de $407 \mathrm{~kg}$ équivalent carbone » (ADEME, 2007, p. 152). On notera d'ailleurs que les chiffres indiqués pour la consommation de gazole et le tonnage débarqué conduisent à une consommation unitaire de 0,5 litre de gazole par kilo de poisson et à des émissions directes de $0,363 \mathrm{~kg}$ e-C par kilo de poisson (le chiffre de $407 \mathrm{~kg}$ e-C par tonne prend en compte des émissions amont de production et transport du carburant qui représentent $11 \%$ des émissions directes).

Les émissions françaises de gaz à effet de serre ont représenté en 2005, pour la métropole, un total de $544 \mathrm{MT}$ e-CO 2 hors $\mathrm{UTCF}^{6}$ (DGEMP/OE, 2002). La consommation de la pêche française métropolitaine at-

\footnotetext{
${ }^{5}$ Pour la viande, les émissions de méthane des bovins et les émissions de protoxyde d'azote des engrais azotés s'ajoutent aux émissions de $\mathrm{CO}_{2}$ du gazole.

${ }^{6}$ Utilisation des terres, leur changement et la forêt (CITEPA, 2007 et 2009).
}

teint plus de 300 millions de litres de gazole ${ }^{7}$ pour l'année 2005. La combustion du carburant de la pêche représente donc $0,17 \%$ des émissions françaises. Au niveau mondial, la consommation unitaire moyenne de gazole est estimée à 0,62 1/kg (Tyedmers et al., 2005), estimation faite à partir de données relevées dans différentes études et agrégées sur la base de 18 pêcheries mondiales. Il s'agit dans notre travail de situer la consommation unitaire de carburant de la pêche bretonne par rapport à ces estimations moyennes plus globales, en distinguant la technique de pêche («traînant » versus « dormant ») et la taille du navire (en mètres).

\section{Résultats}

La figure 2 présente la consommation unitaire de gazole de la pêche, exprimée en litres de gazole par kilo de poisson, selon les types de navire. Les navires traînants de plus de 12 mètres affichent une forte consommation unitaire comparativement aux trois autres catégories. La production de poisson nécessite une consommation unitaire variable de 2 à 2,7 1/ $\mathrm{kg}$ selon les années et la taille du navire, avec une moyenne de $2,51 / \mathrm{kg}$ en 2005 . La consommation unitaire des traînants de moins de 12 mètres tend à diminuer, pour atteindre en 2005 un ratio de 1,5 1/kg. Une partie de l'énergie utilisée par les navires traînants sert à la traction des chaluts, ce qui alourdit le bilan énergétique. Pour les unités pratiquant les arts dormants, en revanche, la consommation de carburant sert essentiellement au déplacement entre le lieu de pêche et le lieu de débarquement.

Les dormants de moins de 12 mètres maintiennent sur la période un ratio voisin de $1 \mathrm{l} / \mathrm{kg}$. La situation des dormants de plus de 12 mètres surprend tant leur consommation unitaire de carburant est faible (moins de 0,2 l/kg). Cela s'explique par la présence dominante de bolincheurs dans cette catégorie (leur longueur maximale est de 21 mètres pour une moyenne de 16 mètres). Ces unités de pêche utilisent un filet tournant, avec une distance d'accès limitée aux pêcheries (principalement d'espèces pélagiques), dans la mesure où celles-ci sont situées principalement dans la bande côtière. La limitation de l'accès aux ressources, par l'instauration de licences de pêche sur les espèces pélagiques, a permis une augmentation de la production par navire (triplement entre 1994 et 2005 : cf. Fig. 1) qui renforce leur productivité et réduit leur dépendance énergétique (Le Floc'h et al., 2007). La consommation unitaire de carburant varie donc d'un

\footnotetext{
${ }^{7}$ La pêche française en métropole a consommé en 2005 près de 0,3 million de litres de gazole hors navires de plus de 40 mètres, lesquels représentent $15 \%$ de la puissance totale embarquée (Planchot et Daurès, 2008). À l'échelle mondiale, la FAO estime la consommation de carburant à 14 millions de litres (Thébaud et al., 2008).
} 


\section{litre de gazole / $\mathrm{kg}$ de poisson}

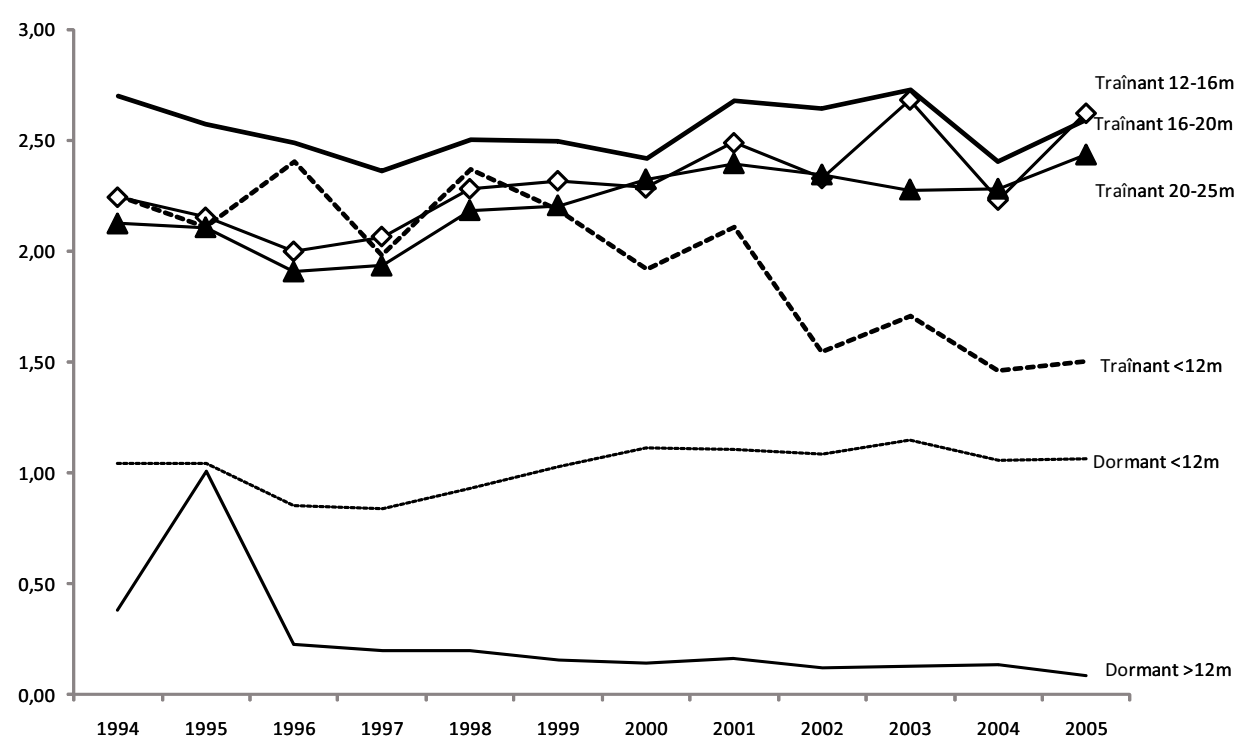

Fig. 2. Évolution de la consommation unitaire de carburant par catégorie de navires (litre par kilo de poisson).

facteur 30 entre les techniques les plus performantes et les moins performantes.

Le ratio moyen, toutes catégories confondues, est de $1,7 \mathrm{l} / \mathrm{kg}$ en 2005. Cette valeur élevée par rapport à la moyenne nationale estimée à $0,5 \mathrm{l} / \mathrm{kg}$ (cf. supra l'estimation de l'ADEME) peut s'expliquer par la plus forte représentation dans l'échantillon des navires traînants, ceux-ci représentant $77 \%$ du tonnage débarqué sur l'ensemble de la période (leur contribution atteignait $90 \%$ en 1994 et $65 \%$ en 2005).

Une autre analyse consiste à calculer la consommation unitaire de carburant en valeur, c'est-à-dire le rapport entre la quantité de carburant et la valeur économique de la production. La figure 3 révèle l'évolution de la consommation de carburant par euro de poisson débarqué au niveau de la première vente sous la halle à marée.

Parmi les «dormants », la supériorité énergétique des plus de 12 mètres, qui était très marquée en volume, l'est nettement moins en valeur. La valeur moyenne débarquée par navire atteint des niveaux comparables à ceux des navires traînants de 16-20 mètres, alors que leur production est proche de celle des navires traînants de 2025 mètres. La plus faible valorisation de la production des dormants de plus de 12 mètres est liée à la nature des espèces capturées, essentiellement des poissons pélagiques (sardine, anchois, maquereau) de faible valeur commerciale. Il en résulte un ratio moyen de 0,17 1/€ en 2005.

Les traînants, qui exploitent des espèces à plus forte valeur marchande (langoustine, sole, baudroie), présentent un ratio variant en 2005 de 0,29 à $0,641 / €$. On y trouve les pêcheries commerciales du golfe de Gascogne, qui se spécialisent dans l'exploitation de ces espèces (Le Floc'h et al., 2008b), même si un phénomène de " glissement de la pêche ${ }^{8}$ » a pu être mis en évidence à l'échelle régionale (Steinmetz et al., 2008). Ce glissement s'opère vers des espèces de niveau trophique inférieur, généralement de petites tailles et de prix unitaires plus faibles.

La consommation unitaire de carburant en valeur varie donc d'un facteur 4 entre les techniques les plus performantes et les moins performantes. Mais les différences observées montrent que, plus que la technique de pêche, c'est la nature des espèces ciblées qui détermine la consommation unitaire de carburant. La technique de pêche apparaît comme un moyen d'atteindre la cible, et la meilleure valorisation des produits les plus recherchés par les consommateurs permet de couvrir des consommations énergétiques plus importantes.

L'intérêt de telles mesures repose sur la traduction de la consommation énergétique en émissions de gaz à effet de serre. Les estimations proposées dans cet article permettent de comparer, en première approximation, les produits de la mer à d'autres sources de protéines : selon les estimations réalisées par l'ADEME, le facteur d'émission de la viande de bœuf est de 4,7 kg e-C par $\mathrm{kg}$ de viande avec os, largement supérieur au porc

${ }^{8}$ Ce phénomène de glissement de l'activité de pêche vers des espèces à bas niveau trophique a été mis en évidence à une échelle mondiale par Pauly et al. (1998). Il conduirait à diriger l'activité d'extraction vers des poissons de plus petite taille dont le prix de marché est tiré vers le bas. Des effets de substitution entre espèces peuvent alors se produire, selon la contribution du secteur dans le marché. Ce type d'effets peut être apprécié par l'étude de la formation des prix et la localisation du prix directeur au sein de la filière (Guillotreau, 2003; Setälä et al., 2008). 


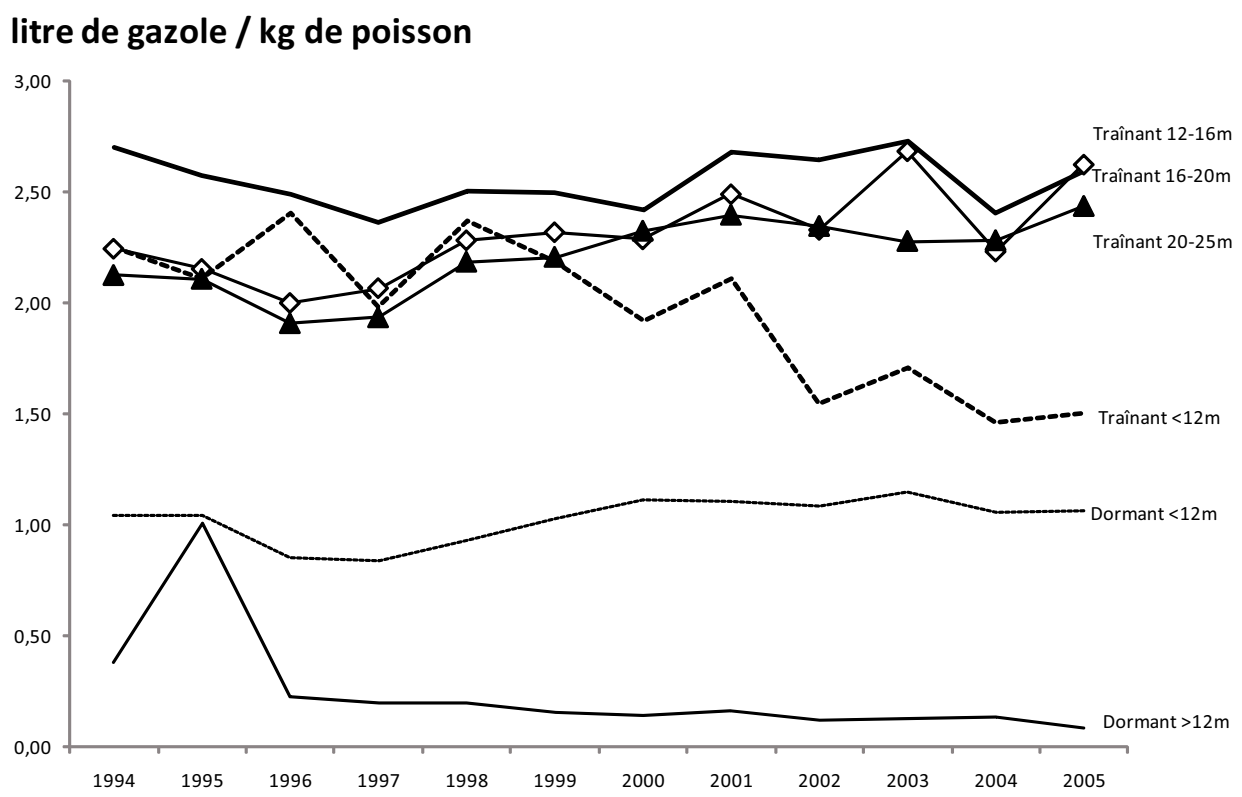

Fig. 3. Évolution de la consommation unitaire de carburant, en valeur marchande, par catégorie de navires (litre par euro).

(1,2 kg e-C par kilo) et au poulet (0,4 kg e-C par kilo). Le poisson débarqué par les dormants a un facteur d'émission de $0,2 \mathrm{~kg}$ e-C par kilo, tandis que celui des traînants a un facteur d'émission de 2,0 kg e-C par kilo, avec une moyenne de $1,4 \mathrm{~kg}$ e-C par kilo de poisson. $\mathrm{Si}$, donc, le poisson est en moyenne comparable au porc, il existe en réalité un écart important entre les poissons tels que la sardine ou le maquereau et les poissons "nobles ", tels que la sole ou la baudroie. Seule une information fine peut permettre aux consommateurs de prendre en compte, dans leurs choix, les facteurs d'émission des produits qui leur sont proposés. Cela montre l'intérêt de mettre en place un système d'information du consommateur, en rendant obligatoire un affichage environnemental suffisamment détaillé, dont les modalités pratiques devront être étudiées par secteur d'activité. De telles mesures font porter sur les consommateurs la responsabilité du choix de produits plus respectueux de l'environnement.

Les émissions de gaz à effet de serre estimées pour les flottilles bretonnes doivent néanmoins être comparées aux chiffres obtenus pour d'autres flottilles européennes (Tab. 2), avant d'être portées à la connaissance des décideurs. La dispersion des résultats soulève alors la question de la pertinence d'un indicateur moyen à une échelle globale (pêcheries mondiales ou même nationales).

Une représentation graphique (Fig. 4) des indicateurs d'intensité énergétique relevés dans les publications scientifiques révèle des valeurs plus élevées dans les études menées à une échelle fine (Marlen, 2009 ; Le Floc’h et Dangeard, présent article) que dans les études reposant sur des données plus globales. Il n'existe aucun doute sur la moindre efficacité énergétique des navires trâ̂nants qui exploitent des pêcheries benthiques (espèces vivant sur le fond) et démersales (espèces vivant à proxi- mité du fond). Seuls trois cas de flottilles pratiquant les techniques traînantes, étudiées à une échelle fine (ibid.), affichent un ratio faible (inférieur à 0,5). Il s'agit alors exclusivement de pêcheries pélagiques. L'étude concernant la pêcherie de morue en Suède (Ziegler et Hansson, 2003) indique que la consommation de carburant par kilo de poisson débarqué s'élève à $1,41 / \mathrm{kg}$ pour les traînants et à 0,34 1/kg pour les dormants. D'autres mesures obtenues pour la Norvège par Schau et al. (2009) montrent des écarts importants entre pêcheries pélagiques $(0,08$ 1/kg pour le sprat européen, de faible valeur commerciale) et pêcheries démersales ou benthiques $(2,45 \mathrm{l} / \mathrm{kg}$ pour la sole de forte valeur commerciale). Thrane (2006) explique le faible ratio de la pêche danoise, égal à $0,131 / \mathrm{kg}$, par un tonnage constitué pour les deux tiers de «poisson industriel » destiné à l'alimentation animale.

On retrouve une cohérence entre nos résultats et ceux produits dans le cadre d'une étude européenne (Marlen, 2009) à une échelle locale. À l'inverse, les estimations menées à une échelle plus globale, voire mondiale (Tyedmers et al., 2005; Hospido et Tyedmers, 2005), affichent de plus faibles intensités énergétiques.

\section{Discussions et perspectives}

Il semble qu'il n'y ait aucun doute sur la prédominance de l'activité de capture comme source principale d'émissions de gaz à effet de serre. Les réponses apportées face à ce constat mettent en avant les avancées technologiques attendues sur la réduction des gaz à effet de serre, notamment par la recherche d'économie dans la consommation de carburant. On peut également suggérer le recours possible à d'autres techniques 
Tableau 2. Indicateurs d'intensité énergétique (litre de carburant par kilo de poisson débarqué) relevés dans la bibliographie.

\begin{tabular}{|c|c|c|c|c|c|c|}
\hline Source & Pays & Année & $\begin{array}{l}\text { Longueur } \\
\text { (mètres) }\end{array}$ & Technique & Pêcherie & $\begin{array}{l}\text { Consommation } \\
\text { unitaire de } \\
\text { carburant }(1 / \mathrm{kg})\end{array}$ \\
\hline Notre étude (présent article) & France & 1994-2005 & $<12$ & Dormant & Démersal & 1,03 \\
\hline Notre étude & France & 1994-2005 & $>12$ & Dormant & Démersal & 0,17 \\
\hline Notre étude & France & 1994-2005 & $<12$ & Traînant & Démersal & 1,98 \\
\hline Notre étude & France & 1994-2005 & $12-16$ & Traînant & Démersal & 2,54 \\
\hline Notre étude & France & 1994-2005 & $16-20$ & Traînant & Démersal & 2,25 \\
\hline Notre étude & France & 1994-2005 & $20-25$ & Traînant & Démersal & 2,18 \\
\hline Marlen, 2009 & Danemark & 2004-2006 & $<12$ & Dormant & & 0,35 \\
\hline Marlen, 2009 & Danemark & 2004-2006 & $12-24$ & Traînant & Démersal & 1,13 \\
\hline Marlen, 2009 & Danemark & 2004-2006 & $24-40$ & Traînant & Démersal & 1,56 \\
\hline Marlen, 2009 & France & $2004-2006$ & $<12$ & Dormant & & 1,1 \\
\hline Marlen, 2009 & France & 2004-2006 & $12-24$ & Traînant & Démersal & 2,4 \\
\hline Marlen, 2009 & Irlande & $2004-2006$ & $<12$ & Dormant & & 1,42 \\
\hline Marlen, 2009 & Irlande & 2004-2006 & $12-24$ & Traînant & Démersal & 1,39 \\
\hline Marlen, 2009 & Irlande & $2004-2006$ & $24-40$ & Traînant & Démersal & 1,74 \\
\hline Marlen, 2009 & Irlande & 2004-2006 & $24-40$ & Traînant & Pélagique & 0,17 \\
\hline Marlen, 2009 & Irlande & $2004-2006$ & $>40$ & Traînant & Pélagique & 0,09 \\
\hline Marlen, 2009 & Pays-Bas & 2004-2006 & $12-24$ & Traînant & Perche & 1,7 \\
\hline Marlen, 2009 & Pays-Bas & $2004-2006$ & $24-40$ & Traînant & Perche & 4,7 \\
\hline Marlen, 2009 & Pays-Bas & 2004-2006 & $>40$ & Traînant & Perche & 3,8 \\
\hline Marlen, 2009 & Belgique & $2004-2006$ & $12-24$ & Traînant & Perche & 3,1 \\
\hline Marlen, 2009 & Belgique & 2004-2006 & $24-40$ & Traînant & Perche & 3,31 \\
\hline Marlen, 2009 & Royaume-Uni & $2004-2006$ & $12-24$ & Traînant & Démersal & 1,3 \\
\hline Marlen, 2009 & Royaume-Uni & 2004-2006 & $24-40$ & Traînant & Démersal & 1,2 \\
\hline Marlen, 2009 & Royaume-Uni & $2004-2006$ & $24-40$ & Traînant & Perche & 3 \\
\hline Marlen, 2009 & Royaume-Uni & $2004-2006$ & $>40$ & Traînant & Démersal & 1,3 \\
\hline Marlen, 2009 & Royaume-Uni & $2004-2006$ & $>40$ & Traînant & Pélagique & 0,1 \\
\hline Tyedmers et al., 2005 & Monde & 2000 & nd & Global & Global & 0,6 \\
\hline Ellingsen et al., 2005 & Norvège & 1980-1999 & nd & Traînant & Démersal & 0,47 \\
\hline Ellingsen et al., 2005 & Norvège & $1980-1999$ & nd & Traînant & Pélagique & 0,07 \\
\hline Thrane, 2006 & Danemark & 2000 & nd & Global & Global & 0,13 \\
\hline Hospido et Tyedmers, 2005 & Espagne & 2003 & $>40$ & Traînant & Seine & 0,43 \\
\hline
\end{tabular}

de capture, relançant le débat sur le retour des techniques (reswitching) mené dans les années 1960 entre deux écoles de pensée en économie ${ }^{9}$. Un retour à des techniques moins intensives en énergie est toutefois contrarié par les politiques conjoncturelles de subvention en période de tensions inflationnistes sur le prix du baril de pétrole (Sumaila et al., 2008; Le Floc'h et al., 2008a). Une autre voie, peut-être plus prometteuse dans la réduction des impacts environnementaux liés aux flottilles de pêche, est celle de l'étiquetage environnemental. L'affichage d'informations destinées aux consommateurs sur l'intensité énergétique pourrait modifier le comportement d'achat. Les consommateurs auraient ainsi l'opportunité de sanctionner rétroactivement les unités de pêche les moins efficaces dans le processus de limitation des gaz à effet de serre.

\footnotetext{
${ }^{9}$ Le débat opposait les deux écoles de Cambridge, l'une américaine dirigée par Samuelson (1962), l'autre britannique (bien que moins identifiée géographiquement) conduite par Robinson (1953) et Sraffa (1960) entre autres.
}

L'expérience accumulée dans le domaine de l'agriculture, en matière d'écobilans des biocarburants, s'avérerait profitable au domaine des pêcheries (Dorin et Gitz, 2008). Cependant, la solution, ici, ne tient pas directement dans la mise en place d'une analyse globale du cycle de vie des produits de la mer. Les résultats présentés dans cet article mettent en question la pertinence d'indicateurs globaux, en particulier lorsque l'information transmise à une échelle trop large ne tient pas compte des techniques de pêche et des espèces ciblées : si un indicateur global a un intérêt à des fins statistiques, ce n'est plus le cas s'il doit être utilisé pour éclairer les choix des consommateurs.

Or, les politiques publiques appliquées à l'échelle nationale ont consisté essentiellement à favoriser les techniques les moins efficaces d'un point de vue énergétique, pénalisées par l'augmentation du coût du carburant. Les réponses apportées par les pouvoirs politiques, indépendamment du cadre européen de la gestion des pêcheries, se sont toujours limitées à des mesures de compensation du prix du carburant. En euro constant sur la base de 


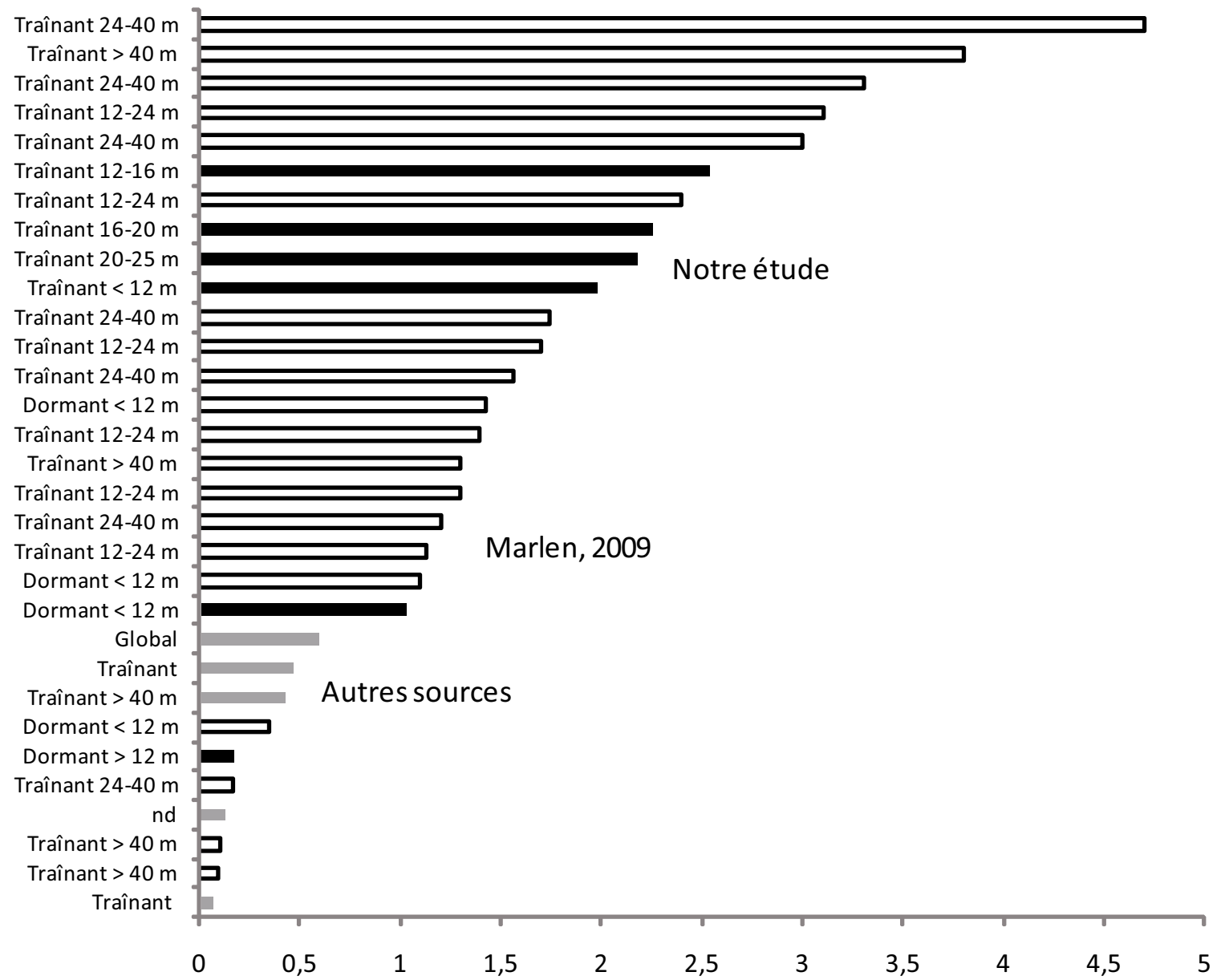

Fig. 4. Valeur des indicateurs d'intensité énergétique (litre de carburant par kilo de poisson débarqué) estimés dans la bibliographie.

l'année 2005, le prix au litre a augmenté de $8 \%$ par an depuis 1994. Sur cette période, on recense deux interventions budgétaires des pouvoirs publics en 2001 et 2005, à la suite de fortes hausses du prix du carburant ${ }^{10}$.

Agissant en sens opposé au principe "pollueurpayeur », ces politiques sont équivalentes à une subvention carbone, alors que la seule tendance compatible avec la maîtrise des gaz à effet de serre est bien l'instauration d'une taxe carbone. Celle-ci irait à l'encontre de toutes les dispositions d'aide à la pêche présentées comme «euro-compatibles ». En ce qui concerne la taxe carbone, dont l'application était envisagée en 2010, une exonération partielle pour les pêcheurs et les agriculteurs avait été prévue (annonce du Premier ministre le 25 septembre 2009). Les politiques publiques continuent donc

\footnotetext{
${ }^{10}$ Les mesures de compensation reposaient sur des allègements des charges sociales et l'exonération de la taxe au débarquement en 2000 et 2001. En 2005 et 2006, elles ont pris la forme d'un financement par l'État du Fonds de prévention des aléas à la pêche. Ces deux mesures de compensation ont été remises en cause par la Commission européenne puisque jugées non compatibles au regard du droit de la concurrence (Union européenne, 2006).
}

à s'inscrire dans un cadre conjoncturel pour soutenir les flottilles de pêche les plus fragilisées par la hausse du coût global (taxes comprises) de l'énergie, au lieu d'envisager des politiques structurelles visant à réduire les intensités énergétiques par des améliorations techniques ou à accompagner l'évolution technologique des flottilles.

Par ailleurs, la question de l'intensité énergétique de la pêche est liée à la question d'une limitation de l'accès aux ressources par quotas ou licences. Un surdimensionnement de la flotte conduit inévitablement à une détérioration du ratio énergétique. La quantité de poisson disponible pour une pêche « raisonnable » est donc limitée, et tout dépassement accentue les problèmes, qu'ils concernent la biodiversité ou les émissions de gaz à effet de serre.

\section{Références}

ADEME, 2007, Bilan Carbone ${ }^{\circledR}$ entreprises et collectivités : guide des facteurs d'émissions, version 5.0. Calcul des facteurs d'émissions et sources bibliographiques utilisées.

Observatoire économique régional des pêches de Bretagne, 2007. Résultats des flottilles artisanales 2005/2006 : note de 
synthèse [contributeurs : Bihel, J., Le Floc'h, P.], Fédération bretonne coopération maritime, Quimper.

CITEPA (Centre interprofessionnel technique d'études sur la pollution atmosphérique), 2007. CITEPA/CORALIE : rapport OMINEA, mise à jour du $1^{\text {er }}$ février 2007, Paris.

CITEPA, 2009. Émissions dans l'air en France métropole: substances relatives à l'accroissement de l'effet de serre, Paris, CITEPA.

Communauté européenne, 2000. Règlement (CE) n 1543/2000 du 29 juin 2000 instituant un cadre communautaire pour la collecte et la gestion des données nécessaires à la conduite de la politique commune de la pêche, Journal officiel des communautés européennes, L176, 1 (http://eur-lex.europa.eu/ JOHtml.do?uri=OJ:L:2000:176:SOM:FR:HTML [page 1]).

Communauté européenne, 2001. Règlement (CE) n 1639/2001 de la commission du 25 juillet 2001 établissant les programmes communautaires minimal et étendu pour la collecte des données dans le secteur de la pêche, Journal officiel des Communautés européennes, L222, 53 (http://eur-lex.europa.eu/JOHtml.do?uri=OJ:L:2001:222: SOM:FR:HTML [page 53]).

DGEMP/OE, 2002. Les équivalences énergétiques et la nouvelle méthodologie d'établissement des bilans énergétiques de la France, Direction générale de l'énergie et des matières premières, ministère de l'Économie, des Finances et de l'Industrie, Paris.

Dorin, B., Gitz, V., 2008. Écobilans de biocarburants : une revue des controverses, Nature Sciences Sociétés, 16, 4, 337-347.

Ellingsen, H., Aanondsen, S.A., 2006. Environmental impacts of wild caught cod and farmed salmon: A comparison with chicken, International Journal of Life Cycle Assessment, 1, 1, 60-65.

Guillotreau, P., 2003. Prices and Margins along the European Seafood Value Chain, Les Cahiers de l'Artemis, 4.

Hospido, A., Tyedmers, P., 2005. Life cycle environmental impacts of Spanish tuna fisheries, Fisheries Research, 76, 174186.

Kline, S., Rosenberg, N., 1986. An overview of innovation, in Landau, R., Rosenberg, N., The Positive Sum Strategy, Washington (DC), National Academy Press, 275-305.

Le Floc'h, P., Daurès, F., Brigaudeau, C., Bihel, J., Thébaud, O., 2007. Analysing fishermen behaviour face to increasing energy costs: A French case study, ICES Annual Science Conference, 17-21 September, Helsinki, Finland.

Le Floc'h, P., Thébaud, O., Boncœur, J., Daurès, F., Guyader, O., 2008a. Une évaluation des performances économiques de la pêche côtière : le cas de la Bretagne, Revue d'économie régionale et urbaine, 5, 753-771.

Le Floc'h, P., Poulard, J.-C., Thébaud, O., Blanchard, F., Bihel, J., Steinmetz, F., 2008b. Analyzing the market position of fish species subject to the impact of long term changes: A case study of French fisheries in the Bay of Biscay, Aquatic Living Resources, 21, 3, 307-316.

Levrel, H., 2008, Les indicateurs de développement durable : proposition de critères d'évaluation au regard d'une approche évolutionniste de la décision, Revue française de socioéconomie, 2, 199-222.
Marlen, B. van (Ed.), 2009. Energy Saving in Fisheries. Final Report, IMARES, Wageningen (http://library.wur.nl/ WebQuery/clc/1901878).

Nations unies, 1992. Convention-cadre des Nations unies sur les changements climatiques. FCCC/INFORMAL/84, GE.0562221 (F) 180705260705 (http://unfccc.int/resource/docs/ convkp/convfr.pdf).

Pauly, D., Christensen, V., Dalsgaard, J., Froese, R., Torres, F., 1998. Fishing down marine food webs, Science, 279, 860-863.

Planchot, M., Daurès, F., 2008. Le Secteur français des pêches maritimes face à l'augmentation du prix du gasoil. Note de synthèse, Système d'informations halieutiques, Ifremer, Plouzané.

Robinson, J., 1953-1954. The production function and the theory of capital, The Review of Economic Studies, 21, 2, 81-106.

Sampson, D.B., 1990. Fishing Technology and Fleet Dynamics: Predictions from a Bio-economic Model. Research Paper 38, Centre for the Economics and Management of Aquatic Resources, Portsmouth (UK).

Samuelson, P.A., 1962. Parable and realism in capital theory: The surrogate production function, The Review of Economic Studies, 29, 3, 193-206.

Schau, E.M., Ellingsen, H., Endal, A., Aanondsen, S.A., 2009. Energy consumption in the Norwegian fisheries, Journal of Cleaner Production, 17, 325-334.

Schau, E.M., Magerholm Fet, A., 2008. LCA studies of food products as background for environmental product declarations, International Journal of Life Cycle Assessment, 13, 3, 255-264.

Setälä, J., Laitinen, J., Virtanen, J., Saarni, K., Nielsen, M., Honkanen, A., 2008. Spatial integration of freshwater fish markets in the Northern Baltic Sea area, Fisheries Research, 92, 196-206.

Sraffa, P., 1960. Production of Commodities by Means of Commodities: Prelude to a Critique of Economic theory, Cambridge (UK), Cambridge Univesity Press.

Steinmetz, F., Thébaud, O., Blanchard, F., Le Floc'h, P., Bihel, J., 2008. A bio-economic analysis of long term changes in the production of French fishing fleets operating in the Bay of Biscay, Aquatic Living Resources, 21, 3, 317-327.

Sumaila, U.R., The, L., Watson, R., Tyedmers, P., Pauly, D., 2008. Fuel price increase, subsidies, overcapacity, and resource sustainability, ICES Journal of Marine Science, 65, 832-840.

Thébaud, O., Daurès, F., Girard, S., Guyader, O., Le Floc'h, P., Le Gallic, B., Montgruel, R., 2008. Données récentes sur la situation économique du secteur de la pêche en France. Document de travail, UMR AMURE, UBO/Ifremer, Plouzané.

Thrane, M., 2006. Life cycle assessment of Danish fish products: New methods and insights, International Journal of Life Cycle Assessment, 11, 1, 66-74.

Tyedmers, P., Watson, R., Pauly, D., 2005. Fueling global fishing fleets, Ambio, 34, 8, 635-638.

Union européenne, 2006. Aide d'État n C 9/2006 (ex-NN 85/2005) - Fonds pour la prévention des risques liées aux activités du secteur de la pêche, Journal officiel de l'Union européenne, C91, 30 (http://eur-lex.europa.eu/JOHtml.do? uri=OJ:C:2006:091:SOM:FR:HTML [page 30]).

Ziegler, F., Hansson, P.-A., 2003. Emissions from fuel combustion in Swedish cod fishery, Journal of Cleaner Production, 11, 303-314. 